\title{
Association Between aquaporin-1 and Endurance Performance: A Systematic Review
}

\author{
Miguel A. Rivera ${ }^{1 *}$ (D) and Thomas D. Fahey ${ }^{2}$
}

\begin{abstract}
Background: There is abundant and mounting information related to the molecular and biological structure and function of the Aquaporin-1 (AQP1) gene and the AQP1-Aquaporin channel. Regulation of water flow across cell membranes is essential for supporting inter- and intracellular fluid balance, which is critical for health and exercise performance. The transmembrane water channel AQP1 is important for cardiorespiratory endurance (CE) because it influences fluid transfers in erythrocytes, endothelial, and pulmonary cells and is vital for transport of ammonium, bicarbonate, carbon dioxide, glycerol, nitric oxide, potassium ion, water, and trans-epithelial and renal water. Very recent publications suggest the association between a DNA sequence variant, rs1049305 (C > G), in the 3'untranslated region of the AQP1 gene and CE performance. Other reports indicate further significant associations between AQP1 channel and CE phenotypes. The purposes of this systematic review were to examine the extent of the associations between the AQP1 rs1049305 genotype and CE exercise performance and body fluid loss in longdistance runners and AQP1 channel associations with other CE phenotypes.

Methods: Data sources: A comprehensive review was conducted using PubMed, EMBASE, CINAHL, and Cochrane electronic databases. The search ranged from January 1, 1988, to December 31, 2018. Studies reported in English, French, and Spanish were considered. Eligibility criteria: The criteria for inclusion in the review were (a) case-control study; (b) unequivocal definition of cases and controls; (c) CE was defined as performance in endurance events, laboratory tests, and/or maximal oxygen consumption; (d) exclusion criteria of known causes; (e) genotyping performed by PCR or sequencing; $(f)$ genotype frequencies reported; and $(g)$ no deviation of genotype frequencies from Hardy-Weinberg equilibrium in the control group. Study appraisal: The systematic review included studies examining the AQP1 gene and AQP1 channel structure and function, associations between the AQP1 gene sequence variant rs1049305 (C > G) and CE performance, body fluid loss in long-distance runners, and other studies reporting on the AQP1 gene and channel CE phenotype associations. Synthesis methods: For each selected study, the following data were extracted: authors, year of publication, sample size and number of cases and controls, CE definition, exclusion criteria, inclusion criteria for cases and controls, methods used for genotyping, genotype, allele frequencies and HWE for genotype frequencies in cases and control groups, and method of AQP1 gene and AQP1 channel analysis.

(Continued on next page)
\end{abstract}

\footnotetext{
*Correspondence: miguel.rivera10@upr.edu; miguelrivera@prtc.net

'Department of Physical Medicine, Rehabilitation \& Sports Medicine, School of Medicine, University of Puerto Rico, Main Building Office A204, San Juan, PR 00936, USA

Full list of author information is available at the end of the article
} 
(Continued from previous page)

Results: The initial databases search found 172 pertinent studies. Of those, 46 studies were utilized in the final synthesis of the systematic review. The most relevant findings were (a) the identification of an independent replication of the association between AQP1 gene sequence variant rs1049305 (C > G) and CE performance; (b) the association of the rs1049305 C-allele with faster CE running performance; (c) in knockout model, using a linear regression analysis of distance run as a function of Aqp1 status (Aqp1-null vs. wild-type mice) and conditions of hypoxia (ambient $\left[\mathrm{O}_{2}\right]=16 \%$ ), normoxia (21\%), and hyperoxia (40\%) indicated that the Aqp1 knockout ran less distance than the wild-type mice $(p<0.001)$; (d) in vitro, a reduced $A Q P 1$ expression was associated with the presence of the rs1049305 G-allele; (e) AQP1 null humans led normal lives and were entirely unaware of any physical limitations. However, they could not support fluid homeostasis when exposed to chronic fluid overload. The limited number of studies with "adequate sample sizes" in various racial and ethnic groups precluding to perform proper in-depth statistical analysis.

Conclusions: The AQP1 gene and AQP1 channel seems to support homeostatic mechanisms, yet to be totally understood, that are auxiliary in achieving an advantage during endurance exercise. AQP1 functions are vital during exercise and have a profound influence on endurance running performance. AQP1s are underappreciated structures that play vital roles in cellular homeostasis at rest and during CE endurance running exercise. The outcome of the present systematic review provide support to the statement of hypotheses and further research endeavors on the likely influence of AQP1 gene and AQP1 channel on CE performance. Registration: The protocol is not registered.

Keywords: Aquaporin 1, Genetics, rs1049305 variant, Distance running, Athletes

\section{Key Points}

- The AQP1 gene and AQP1 channel functions are vital during exercise and have profound influence on cardiorespiratory endurance performance.

- This is significant because genetic (molecular) mechanisms and their effects on cardiorespiratory endurance performance phenotypes are major areas of inquiry in the science and medicine of sport and exercise.

- Regulation of water flow across cell membranes is essential for supporting a proper fluid balance within the cells, which is a critical factor in health status and endurance performance.

\section{Background}

The concept of "genetic mechanisms" and their effect on cardiorespiratory endurance (CE) performance phenotypes is a major area of inquiry in the science and medicine of sport and exercise. CE is the ability of the body to perform prolonged, large-muscle, dynamic exercise at moderate to high levels of intensity [1]. The best clinical and physiological criterion of $\mathrm{CE}$ is the maximal oxygen consumption $\left(\mathrm{VO}_{2 \max }\right)$. The latter is the highest rate at which the body takes up, transports, and uses oxygen at sea level. $\mathrm{CE}$ and the $\mathrm{VO}_{2 \max }$ measures are high, significant, and positively correlated, and both are highly influenced by genetic mechanisms [2]. An intriguing area of research is the relationship between CE exercise performance and molecular genetic mechanisms related to water and solute transport across membranes. The body mass of humans is approximately $70 \%$ water. Regulation of water flow across cell membranes is essential for maintaining an appropriate fluid balance within the cells [3], which is a critical factor in health status and CE performance [4]. A novel finding related to water management at the molecular level occurred in 1988 with the identification of transmembrane water channels in erythrocytes [5]. That work led to a Nobel Prize in Chemistry for Peter Agre in 2003. The capacity of the erythrocytes to take up and release water make them significant in controlling body water distribution throughout the body [6]. The transmembrane water channel protein, which was originally named CHIP28, is now known as Aquaporin-1 (AQP1) [7]. There is abundant and mounting information related to the molecular and biological structure and function of the AQP1 gene and the AQP1 channel [8].

\section{Aquaporin-1 Gene and Channel}

Aquaporins (AQPs) are a family of transmembrane proteins divided into two subfamilies: those which transport only water, and aquaglyceroporins, which transport water and small organic compounds [9]. The AQP1 channel is the best known and most studied of the AQP family. The AQP1 channel is encoded by the AQP1 gene, on chromosome 7 , region p14 [10]. This gene extends $17 \mathrm{~kb}$ pairs and contains four exons and three introns [7]. The AQP1 gene is highly polymorphic, displaying over 150 deoxyribonucleic acid (DNA) sequence variations [11]. The AQP1 channel is a complex, sophisticated, and regulated $28-\mathrm{kDa}$ protein known to 
play several molecular functions and biological processes $[7,12,13]$. The channel shows transmembrane transporter activity for water, ammonium, bicarbonate, carbon dioxide, glycerol, nitric oxide, and renal water. It also shows channel activity for intracellular cGMP-activated cation, and potassium. Other known molecular activity is that of identical protein binding [12]. Moreover, it shows cellular response to cyclic guanosine monophosphate (cAMP), copper ion, dexamethasone stimulus, hydrogen peroxide, organic substance, mercury ions, retinoid acid, salt stress, ultraviolet (UV) light, hyper-osmosis, hypoxia, mechanical stimulus, and stress, also shows a positive regulation of angiogenesis, fibroblast proliferation, and saliva secretion. It negatively regulates cysteine-type endopeptidase activity involved in the apoptotic process. Further roles include secretion of cerebrospinal fluid and pancreatic juice, cGMP biosynthetic process, establishment or maintenance of actin cytoskeleton polarity, chondrocyte water homeostasis, lateral ventricle development, maintenance of symbiontcontaining vacuole by host, and multicellular organismal water homeostasis [12].

The AQP1 channel exists as a tetramer with each subunit containing its own functionally independent pore $[14,15]$. Each subunit contains six transmembrane helices packed to form a trapezoid-like structure [16]. The pore's dumbbell-like shape consists of an extracellular area, a selectivity filter containing the constriction region, and a cytoplasmic area. The water permeability and the ion conductance do not occur through the same pathway [17], as the individual subunit pore transports water and the central pore acts as a selective ion channel that allows the flow of ions and water [18]. The water selectively of AQP1 is caused by the size exclusion effect $[19,20]$. The narrow or mid-region at the channel imposes a strict restriction, so there is only one water molecule at a time allowed to pass through [19]. The flux of water is at approximately $10^{9}-10^{10}$ molecules per second per channel, with an activation energy as low as the one associated with the self-diffusion rate in bulk water [19, $21,22]$. When coming close to the mid-region, the water molecule undergoes a rotation helping to form hydrogen bonds between its oxygen and specific amino groups [23]. This rotation and its effect is essential for proton exclusion [24]. The monomeric pores of AQP1 have strong hydrogen bonds between the protein and water molecules inside the channel; this makes the monomers less gas permeable than the central pore [25]. Recent evidence suggests that the hydrophobic central pore of AQP1 is permeable to both $\mathrm{CO}_{2}$ and $\mathrm{O}_{2}$ [25]. Regulating cation permeability of AQP1 through a cGMPdependent signaling cascade has significant importance to the control of secretion and absorption of fluid, and regulation of cell volume in many tissues that highly express this channel [18]. AQP1 has been identified in various tissues, including red blood cells, endothelial cells, and smooth, skeletal, and cardiac muscles [26, 27]. The major aquaporin of the cardiovascular system is AQP1, which probably regulates water permeability of the heart's capillary networks by mediating the flow of water through the endothelial layer into the blood. Very recent publications [28-30] suggest the association between a DNA sequence variant, rs1049305 ( $\mathrm{G}>\mathrm{C}$ ), in the $3^{\prime}$ - untranslated region ( 3 ' UTR) of the $A Q P 1$ gene and the CE performance level and body fluid loss in long-distance runners. Other reports [31, 32] indicate further significant associations between AQP1 and CE phenotypes. To the best of our knowledge, no in-depth reviews examining, describing, or analyzing such AQP1 associations have been published. There is consensus that individual reports do not provide sound and clear evidence as that provided by systematic reviews. Therefore, the purpose of this systematic review was to answer the question of the extent of the association between the AQP1 rs1049305 ( $\mathrm{G}>\mathrm{C}$ ) genotype and CE performance level and body fluid loss in long-distance runners, along with other studies reporting on the AQP1 and CE phenotypes associations. The outcome of the present systematic review could provide support to further research endeavors and statement of hypotheses on the possible influence of $A Q P 1$ gene and channel on $\mathrm{CE}$ performance.

\section{Methods}

\section{Eligibility criteria}

The search examined papers published between January 1, 1998, and December 31, 2018. No limits were applied to searches and studies reported in English, French, and Spanish. The review included studies examining the $A Q P 1$ gene and AQP1 channel structures and physiology potentially underlying $\mathrm{CE}$, association between the $A Q P 1$ gene sequence variant at reference single-nucleotide polymorphism cluster identification number (rs) 1049305 (C > G) and CE performance, and body fluid loss in long-distance runners and studies reporting on the $A Q P 1$ gene and channel associations with other $C E$ phenotypes.

\section{Inclusion criteria}

The criteria to include in the review were (a) case-control study; (b) unequivocal definition of cases and controls; (c) CE defined as performance in endurance events (preferably running events), laboratory tests, and/or $\mathrm{VO}_{2 \max }$; (d) exclusion criteria of known causes; (e) genotyping performed by the polymerase chain reaction (PCR) or sequencing; (f) genotype frequencies reported; (g) no deviation of genotype frequencies from HardyWeinberg equilibrium (HWE) in the control group; and 
(h) AQP1 gene and channel structures and functions studies potentially underlying $\mathrm{CE}$.

\section{Information sources}

A comprehensive review of the literature was conducted using PubMed, Excerpta Medica database (EMBASE), Cumulative Index of Nursing and Allied Health Literature (CINAHL), and Cochrane electronic databases detailing papers published between January 1, 1998, and December 31, 2018

\section{Search strategy}

For each electronic database search, the following keywords were used Aquaporin-1, Aquaporin-1 gene structure, Aquaporin-1 channel structure + function, $A Q P 1$, AQP1, AQP1 + polymorphism + rs1049305, cardiorespiratory + endurance, body fluid loss, association studies, case-control, polymorphism, single-nucleotide polymorphisms (SNPs), $\mathrm{VO}_{2 \max }$, cardiovascular fitness, cardiorespiratory fitness, aerobic fitness, aerobic capacity, longdistance running, and body water regulation. To reduce the risk of missing studies, the reference lists of the retrieved articles were also examined, along with grey literature (produced at all levels of government, academics, business, and industry in print and electronic formats, but which are not controlled by commercial publishers) [33] or sources other than peer-reviewed journals. If merited, corresponding authors were contacted for additional information on published and unpublished studies. The authors performed the literature search independently. The retrieved publications were deposited in an electronic file (Dropbox: Dropbox Inc., San Francisco, CA) which was accessible to both authors. That avoided duplications and allowed each author to rapidly evaluate incoming documents. The authors first examined the article title for relevance to the topic and probable link to the inclusion criteria. If the title was not clear regarding the above, both authors reviewed the abstracts. When abstracts were relevant, the full text of the article was reviewed. Any disagreements were discussed and resolved by consensus.

\section{Data collection process}

The authors extracted data items and entered them on an Excel spreadsheet. For each finally selected study, the following data were extracted: authors; year of publication; type of study; $A Q P 1$ gene details; AQP1 channel details; population and number of cases and controls; CE definition; exclusion criteria; inclusion criteria for cases and controls; methods used for genotyping, genotype, and allele frequencies; and HWE for genotype frequencies in cases and control groups. If HWE was not informed, it was calculated by the authors. The search items were based on established terminology using
Cochrane definitions where possible. Corresponding authors were contacted for other relevant information. Appropriate data extraction was corroborated by each investigator.

\section{Risk of bias in individual studies}

The main risk of bias in individual studies is that genotyping methods differ between studies.

\section{Summary measures}

Summary measures included genotype and allele frequencies in cases and controls, chi-square analysis of genotype and allele frequencies in cases and controls, odds ratios, chi-square analysis of genotype by $\mathrm{C}$-allele carrier status and allele frequencies in cases and controls, $A Q P 1$-null vs wild-type phenotypes contrasts, performance level by genotype schemes, and absolute contrast between the $\mathrm{C}$-allele frequency prevalence in different ethnic and racial groups.

\section{Synthesis of results}

This involves judgment of study results by authors and how results conformed to the hypothesis dealing with $A Q P 1 / \mathrm{AQP} 1$ and CE performance.

\section{Results \\ Outcome of Scrutiny}

Figure 1 shows a flow chart of the studies' selection process. The initial database search found 172 pertinent studies. A further screening of those 172 studies led to the exclusion of 118 publications due to marginal relevance and the retention of 54 documents for eligibility determination. That process led to the rejection of eight studies due to non-coincidence in biological (structural and functional) traits of the AQP1 channel. The final outcome yielded 46 studies that were utilized in the synthesis of the present systematic review.

\section{AQP1 Case-Control and CE Performance}

In humans (see Table 1), the first line of evidence to support the hypothesis of association between the AQP1 gene and $C E$ performance was provided by Martinez et al. [28]. That report was an observation study using a genetic epidemiology model in a case-control design. They examined the association between a DNA sequence variant, rs1049305 (C > G), in the 3' UTR of the $A Q P 1$ gene and CE performance level in male and female Hispanic marathon runners $(n=784)$. Cases (fast runners; $n=396$; men $=225$; women $=171$ ) were finishers in the top third percentile for their age and sex, while controls (slow runners; $n=388$; men $=221$; women $=167)$ finished in the lowest third percentile. The reported genotype frequencies were in HWE $\left(X^{2}, p\right.$ $\geq 0.05)$ and were not significantly $\left(X^{2}, p \geq 0.05\right)$ different 


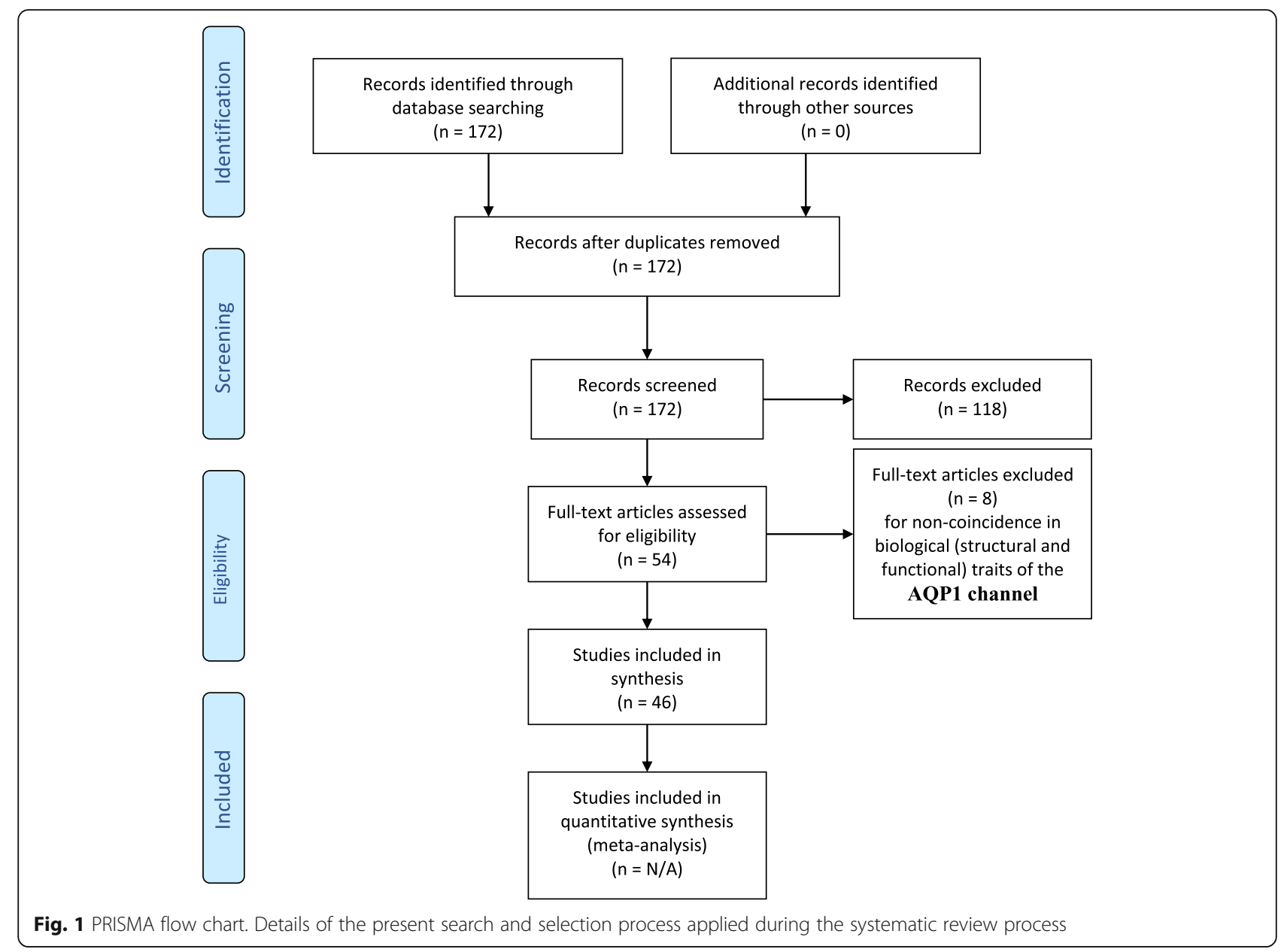

between sexes. Given there were similar genotypic frequency distributions in men and women, for both cases (fast runners) and controls (slow runners), the data for both sexes were pooled. Chi-square test on the pooled data revealed a significant $\left(X^{2}=6.94, p=0.03\right)$ difference in the genotype prevalence distribution between the cases (fast runners) and controls (slow runners).

The observed allelic frequency distributions within the cases (fast runners) and controls (slow runners) revealed no sex differences $\left(X^{2}, p \geq 0.05\right)$. The sex-pooled allelic frequency distribution revealed significant differences $\left(X^{2}=\right.$ $7.55, p=0.005$ ) between cases (fast runners) and controls (slow runners). In both sexes, within cases and controls, the $\mathrm{C}$-allele was the less frequently observed. The calculated odds ratio $=1.35$ and its $95 \%$ confidence interval $(\mathrm{CI})$ $(1.08-1.67)$ suggested that the $C$-allele was more likely $(p=$ $0.005)$ prevalent in the cases (fast runners) than in the controls (slow runners). It is noteworthy that the $A Q P$ lgene rs1049305 $(\mathrm{C}>\mathrm{G})$ is in the 3' UTR. The 3' UTR of messenger RNA has been associated with regulating gene expression [34]. The 3' UTR controls the nuclear export, sub-cellular targeting, and rates of translation and degradation of DNA. The genes controlled by the sequence of the
3' UTR are generally regulatory proteins, and their irregular expression may have serious effects on humans [35].

\section{AQP1 Channel and CE Performance}

$\mathrm{Xu}$ et al. [32], using mice, provided evidence for an association between the Aqp1 channel and CE performance. They tested the hypothesis that the Aqp1 channel plays a physiologically influential role in $\mathrm{O}_{2}$ transport, since the Aqp1 channel is present at high levels in erythrocytes and the pulmonary capillary endothelium. They compared voluntary wheel running over a 24 -h period in Aqp1-null vs. wild-type mice under conditions of hypoxia (ambient $\left[\mathrm{O}_{2}\right]=16 \%$ ), normoxia $(21 \%)$, and hyperoxia (40\%). Linear regression analysis of distance run as a function of Aqp1 status and $\left[\mathrm{O}_{2}\right]$ treating $\left[\mathrm{O}_{2}\right]$ categorically referring to $21 \% \mathrm{O}_{2}$ indicated that the Aqp1 knockout reduced the distance run by $4.7 \pm 0.5 \mathrm{~km}(p<$ 0.001 ), adjusting for $\left[\mathrm{O}_{2}\right]$. Compared to $21 \% \mathrm{O}_{2}$, reducing $\mathrm{O}_{2}$ to $16 \%$ reduced the distance run by $1.6 \pm 0.6$ $\mathrm{km}(p=0.01)$, while increasing $\mathrm{O}_{2}$ to $40 \%$ increased the distance run by $1.2 \pm 0.6 \mathrm{~km}(p=0.04)$, adjusting for Aqp1 status. These findings led to the conclusion that the Aqp1-null mice have a major effect in voluntary 

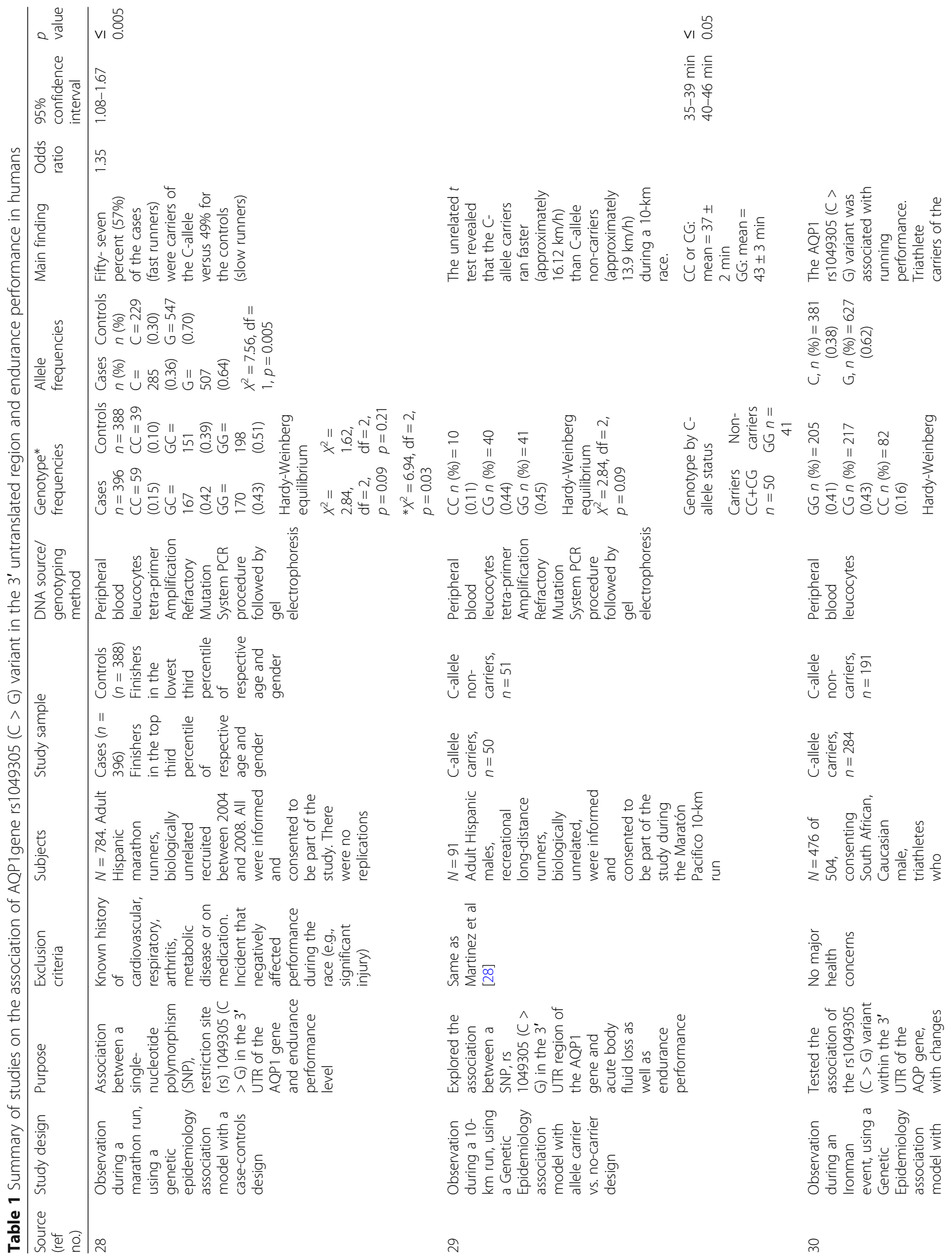

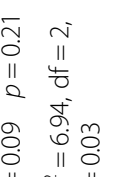

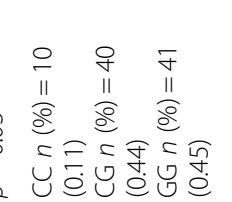

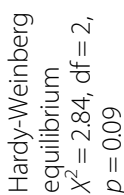

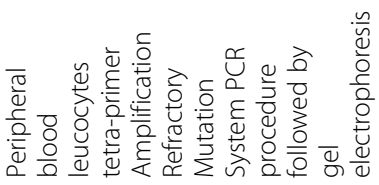

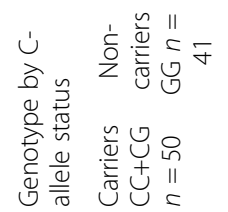

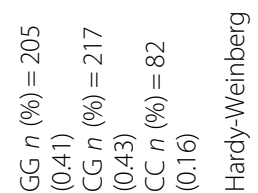

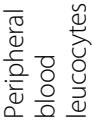

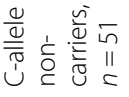

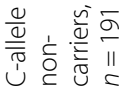

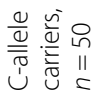

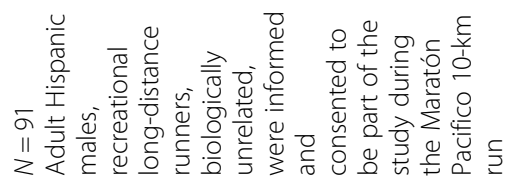

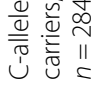

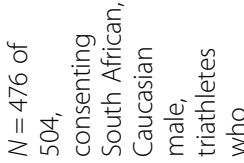

$\sqrt[\pi]{\frac{\pi}{4}}$

$\approx \stackrel{0}{*}$

㝕亮怘

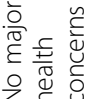

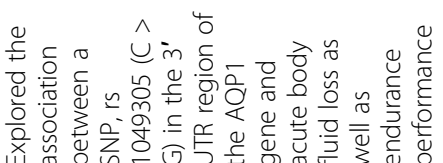

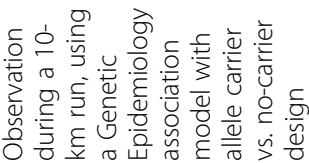

న

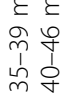

$$
\text { 量 }
$$




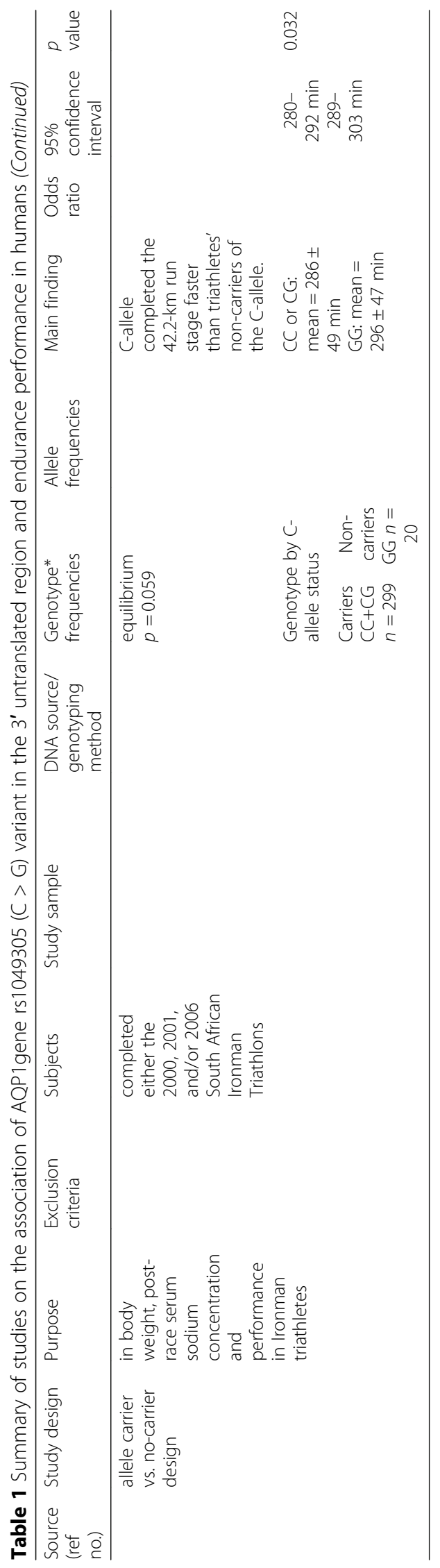


exercise tolerance (CE performance), consistent with the hypothesis that Aqp1 plays an important physiological role in $\mathrm{O}_{2}$ transport across plasma membranes. It is well accepted that in humans the execution of prolonged exercise (like distance running) depends highly on molecular mechanisms mostly related to the management of $\mathrm{O}_{2}$.

\section{AQP1 Channel and CE Performance Correlates}

The present observational study of the AQP1 channel shed further light on the possible role of a molecular mechanism, like that related to the AQP1 channel presence or absence and the acute response to exercise and $\mathrm{O}_{2}$ management. In humans, prolonged exercise capacity as that required by long-distance running is highly influenced by $\mathrm{VO}_{2 \max }$, metabolic economy, lactate threshold, temperature regulation, and fatigue resistance. Abundant information indicates the genetics mediate the magnitude of these mechanisms [2]. Of those five factors, the primary determinant of endurance exercise performance is the $\mathrm{VO}_{2 \max }$ [36]. One of the strongest arguments for such contention is that endurance performance and $\mathrm{VO}_{2 \max }$ are strongly and positively associated. New findings [37] arising from a systematic review of 15 studies and meta-analysis indicated that the weighted means for heritability of $\mathrm{VO}_{2 \max }$ absolute values and those adjusted for body weight and for fat-free mass were 0.68 (95\% CI 0.59-0.77), 0.56 (95\% CI 0.47-0.65), and 0.44 (95\% CI $0.13-0.75)$, respectively. The meta-regression analysis revealed that sex could partially explain the heterogeneity in the $\mathrm{VO}_{2 \max }$ heritability estimates adjusted by body weight. The heritability estimates reported among the studies were statistically significant. Last, for submaximal endurance, phenotypes and endurance performance heritabilities were 0.49 (95\% CI $0.33-0.65$ ) and 0.53 (95\% CI $0.27-0.78$ ), respectively.

\section{AQP1 C-Allele Carrier Status and CE Performance}

In humans, the second line of support for the hypothesis of an association between the $A Q P 1$ gene and CE performance was shown by Rivera et al. [29] (see Table 1). For a second time, an observational study using a genetic epidemiology model evaluated the association between the DNA sequence variant, $\operatorname{rs} 1049305(C>G)$, in the 3' UTR of the $A Q P 1$ gene and the CE performance-related phenotype. In this occasion, elapsed running time in a $10-\mathrm{km}$ event was compared by $A Q P 1 \mathrm{C}$-allele carrier status, e.g., carriers (homozygous for $\mathrm{C}$-allele $(\mathrm{CC})$ and heterozygous for $C$-allele (CG); $n=50$ ) and non-carriers (homozygous for G-allele (GG); $n=41$ ). The main findings indicated that $A Q P 1 \mathrm{C}$-allele carries ran an average of $13.4 \%$ faster $(p<0.05)$ than non-carriers during the $10-\mathrm{km}$ race, which is approximately $16.12 \mathrm{~km} / \mathrm{h}$ for carriers and $13.9 \mathrm{~km} / \mathrm{h}$ for non-carriers. There was no difference in training status between the two groups (carriers vs non-carriers of the $A Q P 1 \mathrm{C}$-allele). These findings provide further support to the notion that interindividual variability in CE performance could be partly explained by molecular mechanisms, such as DNA sequence variations. The findings of Rivera et al. [29] provide additional support to those of Martinez et al. [28], suggesting the participation of $A Q P 1$ rs1049305 CC and CG genotype in promoting endurance running performance level.

In humans (see Table 1), a third line of evidence provided further support for the possible role of $A Q P 1$ genotype in $\mathrm{CE}$ performance. This time, the association between CE performance and the $\operatorname{rs} 1049305(\mathrm{C}>\mathrm{G})$ variant within the $3^{\prime}$ UTR region of the $A Q P 1$ gene was evaluated in South African Caucasian male $(n=504)$ finishers in either the $2000(n=112), 2001(n=222)$, and $2006(n=170)$ South African Ironman Triathlons [30]. Their results replicated those of Martinez et al. [28] and Rivera et al. [29] by reporting that the AQP1 rs1049305 $\mathrm{C}$-variant was associated with the duration of marathon running segment in three Ironman events. Triathletes who carried the C-allele completed the $42.2-\mathrm{km}$ run stage faster (mean 286, $s=49 \mathrm{~min}$ ) than triathletes with the GG genotype (mean 296, $s=47 \mathrm{~min} ; P=0.032$ ). That study also contended that their findings and those of Martinez et al. [28] and Rivera et al. [29] are not predictors of endurance performance but are evidence that the AQP1 rs1049305 C-variant contributes to a physiological state receptive to training and beneficial to endurance (long distance) running performance. Some further argue that the weakness of observing a similar genotype effect on performance in the swim and bike stages likely reflects the differing physiological requirements of these activities [30].

\section{Expression, In Vitro, and AQP1 G Allele}

One report [38] revealed, that in vitro (see Table 2), a reduced AQP1 expression was associated with the presence of the rs1049305 G-allele. It was postulated that such reduction in expression of AQP1 could be attributed to an increase in the binding affinity of a microRNA-129 precursor to its binding site two base-pairs (bp) away from the rs1049305 [38]. The same study indicated that with liver fibrosis patients, the AQP1 rs1049305 CC genotype was associated with lower serum sodium concentration and lower serum osmolality when compared to patients with a CG or GG genotype. Saunders et al. [30] hypothesized that reductions in the expression of AQP1 in the presence of the G-allele could cause a slower response to changes in osmotic gradient during exercise. That notion substantiated by the Tam and Noakes [4] observation that serum osmolality is physiologically defended during exercise. 


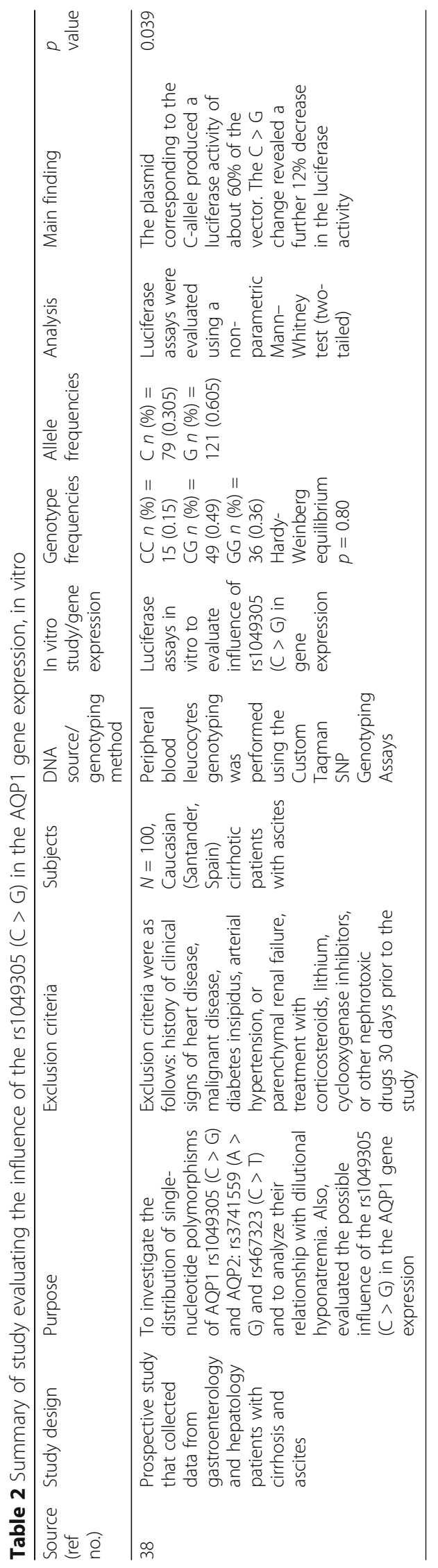




\section{Prevalence of AQP1 C Allele}

Kenyan and Ethiopian runners have dominated Olympic middle- and long-distance running events since the 1968 games in Mexico City [39]. The population distribution of the $A Q P 1$ gene $\mathrm{C}$-allele might partially explain this phenomenon. A report from the National Center for Biotechnology Information [40] found small variations in the frequency (\%) of the $A Q P 1 \mathrm{C}$-allele between Europeans $(0.30 \%)$, Asians $(0.38 \%)$, and Caucasians $(0.42$ $\%)$, but a striking prevalence of the $A Q P 1 \mathrm{C}$-allele in African Americans (0.86 \%) and Sub-Saharans (0.98\%). Others [28] reported that in Hispanics the prevalence of the C-allele was $0.36 \%$ in fast runners (cases) and 0.30 $\%$ in slow runners (controls).

\section{AQP1 Channel Activity Under Hypoxic Exercise}

Huang and Wang [31] used a different approach to the study of $A Q P 1$ gene and endurance exercise. They examined the effects of aerobic interval training (AIT) and moderate continuous training (MCT) on osmotic stressmediated rheological function and AQP1 channel activity of human erythrocytes under hypoxic exercise (HE) stress in humans. Thirty healthy sedentary males were randomly assigned to either the AIT group which performed 3-min intervals at $40 \%$ and $80 \% \mathrm{VO}_{2 \max }, n=15$, or the MCT group required to perform sustained exercise at $60 \% \mathrm{VO}_{2 \max }, n=15$, for $30 \mathrm{~min} /$ day, 5 days/week for 6 weeks. Erythrocyte rheological responses to HE $\left(100 \mathrm{~W}\right.$ under $12 \% \mathrm{O}_{2}$ for $30 \mathrm{~min}$ ) were determined before and after various regimens. The findings revealed that acute HE increased osmotic fragility and decreased deformability of erythrocytes, and depressed erythrocyte AQP1 activity as indicated by increased magnesium chloride $\left(\mathrm{HgCl}_{2}-\right)$ induced instability of erythrocyte membrane under hypotonic conditions. After the 6 weeks of exercise intervention, the AIT group exhibited higher maximal power output and $\mathrm{VO}_{2 \max }$ than the MCT group. Both AIT and MCT diminished the extents of enhanced osmotic fragility, reduced deformability, and AQP1 activity of erythrocytes caused by HE. They concluded that AIT was superior to MCT for enhancing aerobic capacity. Either AIT or MCT effectively alleviated the impairments of erythrocyte rheological characteristics and AQP1 function evoked by HE.

\section{AQP1 and Body Fluid Loss During Exercise}

Along these lines, Rivera et al. [29] and Saunders et al. [30] examined body fluid loss (weight changes) association with the $A Q P 1$ rs1049305 $(C>G)$ variant during endurance runs. Rivera et al. [29] reported that during a $10-\mathrm{km}$ road race, carriers of the AQP1 rs1049305 C-allele had a greater adjusted body fluid loss $(3.7 \pm 0.9 \mathrm{~kg})$ than non-carriers $(1.5 \pm 1.1 \mathrm{~kg})(P<0.05)$. Saunders et al. [30] reported no genotype effect on absolute body weight changes in response to the 42-km running segment of Ironman Triathlons. The latter study argued that the observed opposing findings were attributed to methodological issues [30]. In the Ironman Triathlon study of Saunders et al. [30], before and after absolute values of body weight were used as indicators of body fluid loss. Conversely, Rivera et al. [29] determined body fluid loss from the difference between nude body weight (weight before $10 \mathrm{~km}$ - weight after $10 \mathrm{~km}$ ) with adjustments for fluid intake, respiratory water loss, and urine excretion. Tam and Noakes [4] reviewed the literature pertaining to the controversy of when and why absolute body weight should be adjusted, given practical and scientific endeavors. It is beyond the present review to go further into such controversy.

The exercise-induced body fluid loss differences, by AQP1 genotype, observed by Rivera et al. [29] may also indirectly explain the AQP1 association with running performance. The observation that a high body fluid loss is associated with faster running performance in endurance events is not an isolated event. As found by Saunders et al. [30], triathlete finishers of the 2000, 2001, and 206 South African Ironman Triathlon who lost the most body weight during the whole race had better (faster) finishing times than triathletes who lost less body weight. In addition, others had reported significant inverse correlations between body weight changes because of participation in a $100-\mathrm{km}$ ultramarathon $(n=$ 50; $r=-0.31 ; p=0.023$ ). Faster runners lost more body mass compared with slower runners while also drinking more [41].

A relevant finding of this systematic review is that during osmotic stress, such as intense exercise [26, 42], AQP1 facilitates the transfer of water from blood into muscle via rapid trans-epithelial transport [43], assists in body fluid balance in various systems, provides osmotic protection, and serves as a conduit for water reabsorption and thermal control [14, 42]. The AQP1 channel, due to its known biological functions, could promote cellular homeostasis during intense exercise by action on nitric oxide and $\mathrm{CO}_{2}$ transport [12], two factors linked to endurance performance and prolonged exercise [6, 29]. Wakayama [44] hypothesized that AQP1 might speed skeletal muscle regeneration because of its role in enhancing intramuscular endothelial function. Athletes with the more active AQP1 gene $\mathrm{C}$-allele might train harder and recover faster [29]. More active AQP1 channels in the skeletal muscle and sweat glands might provide several advantages in endurance athletes. They might promote cooling via increased convective heat transfer and sweat rate $[26,45,46]$. Sugie et al. [6] found that AQP1s in erythrocytes were critical for body water management throughout the body. 


\section{AQP1 Null Individuals}

In humans, AQP1 null individuals led normal lives and were entirely unaware of any physical limitations [14]. However, they could not maintain fluid homeostasis when exposed to subacute or chronic fluid overload.

\section{Discussion}

The central finding of the present systematic review was the independent replication of the association between AQP1 gene sequence variant $\mathrm{rs} 1049305(\mathrm{C}>\mathrm{G})$ in the 3' UTR and CE performance [28-30]. Further support to the likelihood that the $A Q P 1$ gene and channel are related to $C E$ performance was substantiated by peerreviewed publications $[6,14,17,32,38,40,42-44]$. Successful performance in endurance running is a complex, multi-factorial trait, also known as phenotype. A phenotype (e.g., endurance running performance) is the ultimate physical expression of DNA and is caused by the interaction of many proteins evolving from DNA. Such processes can get complicated. Thousands of genes create thousands of different proteins that later interact in complex ways, influenced by diet, training, and environmental/ambient conditions. Performance is dictated by the interaction between environment and the intrinsic genetic factors arising from our individual DNA. Basically, each gene can produce a single protein, which can take several forms. The AQP1 gene rs1049305 (C > G) have three forms: CC, CG, and GG. The present systematic review findings favor the hypothesis that $\mathrm{CC}$ and CG forms are apparently positive contributors to the complex physiological state receptive to training and beneficial to $C E$ (long distance) running performance level, while other form of the gene (GG) was seemingly less functional or poorly associated [28-30].

\section{Limitations}

The main limitation of the present review was the limited number of studies with "adequate sample sizes" in various racial and ethnic groups precluding to perform proper in-depth statistical analysis.

\section{Conclusions}

The $A Q P 1$ gene rs1049305 C-allele appears to provide for the enhancement of homeostatic mechanisms, yet to be understood, that are auxiliary in achieving an advantage during $\mathrm{CE}$ running exercise. The $\mathrm{C}$-allele seems to allow for a greater fluid loss and fluid intake in fast versus slow runners. Perhaps, the AQP1 gene rs1049305 Callele and the AQP1 channel might facilitate more intense training, faster recovery, and enhanced temperature regulation. Greater AQP1 [14] activity in erythrocytes with the rs $1049305 \mathrm{C}$-variant allows greater fluid distribution throughout the body, while promoting cellular homeostasis and $\mathrm{CO}_{2}$ transport. AQP1s are underappreciated structures that play vital roles in cellular homeostasis at rest and during CE running exercise.

The AQP1 gene and channel seems to support homeostatic mechanisms, yet to be totally understood, that are auxiliary in achieving an advantage during endurance running exercise. AQP1 functions are vital during exercise and have a profound influence on endurance running performance.

\section{Abbreviations \\ 3' UTR: 3' Untranslated region; AIT: Aerobic interval training; AQP1: Aquaporin-1 channel; AQP1: Aquaporin-1 gene; AQPs: Aquaporins; CC: Homozygous for C-allele; CE: Cardiorespiratory endurance; CG: Heterozygous for C-allele; CGMP: Cyclic guanosine monophosphate; Cl: Confidence interval; CINAHL: Cumulative Index of Nursing and Allied Health Literature; $\mathrm{CO}_{2}$ : Carbon dioxide; DNA: Deoxyribonucleic acid; EMBASE: Excerpta Medica database; GG: Homozygous for G-allele; HE: Hypoxic exercise stress; $\mathrm{HgCl}_{2}$-: Magnesium chloride; HWE: Hardy- Weinberg equilibrium; MCT: Moderate continuous training; $\mathrm{O}_{2}$ : Oxygen; PCR: Polymerase chain reaction; rs: Reference single nucleotide polymorphism cluster identification number; SNPS: Single nucleotide polymorphisms; UV: Ultraviolet; $\mathrm{VO}_{2 \text { max }}$ : Maximal oxygen consumption}

\section{Acknowledgements}

Not applicable

\section{Authors' Contributions}

Both authors contributed in equal proportion in all phases of the study. Any disagreements were discussed and resolved by consensus. All authors read and approved the final manuscript.

\section{Funding}

The authors have no funding sources to disclose.

\section{Availability of Data and Materials}

This study examined published results in each cited reference. All references are readily available in public search engines.

Ethics Approval and Consent to Participate

Not applicable

Consent for Publication

Not applicable

\section{Competing Interests}

The authors, Miguel A. Rivera and Thomas D. Fahey, declare that they have no competing interests.

\section{Author details}

${ }^{1}$ Department of Physical Medicine, Rehabilitation \& Sports Medicine, School of Medicine, University of Puerto Rico, Main Building Office A204, San Juan, PR 00936, USA. ²Department of Kinesiology, California State University, Chico 95929-0330, CA, USA.

Received: 30 April 2019 Accepted: 19 August 2019

Published online: 05 September 2019

\section{References}

1. Bassett DR, Howley ET. Limiting factors for maximum oxygen uptake and determinants of endurance performance. Med Sci Sports Exerc. 2000;32(1): 70-84.

2. Bouchard C, Wolfarth B, Rivera MA, Gagnon J, Simoneau J-A. Genetic determinants of endurance performance. In: Shephard RJ, Åstrand PO, editors. The Encyclopaedia of Sports Medicine, Genetic and Molecular Aspects of Sports Performance. Volume 18 of The Encyclopaedia of Sports Medicine. XVIII: Oxford: Wiley-Blackwell Scientific; 2011. p. 223-242.

3. Knepper MA, Kwon T-H, Nielsen S. Molecular physiology of water balance. $\mathrm{N}$ Engl J Med. 2015;372:1349-58. 
4. Tam N, Noakes TD. The quantification of body fluid allostasis during exercise. Sports Med. 2013;43(12):1289-99.

5. Denker BM, Smith BL, Kuhajda FP, Agre P. Identification, purification, and partial characterization of a novel Mr 28,000 integral membrane protein from erythrocytes and renal tubule. J Biol Chem. 1988;263(30):15634-42.

6. Sugie J, Intaglietta M, Sung LA. Water transport and homeostasis as a major function of erythrocytes. Am J Physiol Heart Circ Physiol. 2018;314(5):H1098H107

7. Moon C, Preston GM, Griffin CA, Jabs EW, Agre P. The human aquaporinCHIP gene. Structure, organization, and chromosomal localization. J Biol Chem. 1993;268(21):15772-8.

8. The UniProt Consortium. UniProt: the universal protein knowledgebase. Nucleic Acids Res. 2017:45(D1):D158-D69.

9. Hub JS, de Groot BL. Mechanism of selectivity in aquaporins and aquaglyceroporins. Proc Natl Acad Sci USA. 2008;105(4):1198-203.

10. Deen PMT, Weghuis DO, Geurs van Kessel A, Wieringa B, van Os CH. The human gene for water channel aquaporin 1 (AQP1) is localized on chromosome 7p15-->p14. Cytogenet Cell Genet. 1994;65(4):243-6.

11. Stelzer G, Rosen N, Plaschkes I, Zimmerman S, Twik M, Fishilevich S, et al. The GeneCards Suite: from gene data mining to disease genome sequence analyses. Curr Protoc Bioinform. 2016;54:1):1.30.1-1..3.

12. Breuza L, Poux S, Estreicher A, Famiglietti ML, Magrane M, Tognolli M, et al. The UniProtKB guide to the human proteome. J Biol Databases Curation. 2016; Published online 2016 Feb 19.

13. Zhang W, Zitron E, Homme M, Kihm L, Morath C, Scherer D, et al. Aquaporin-1 channel function is positively regulated by protein kinase $C$. J Biol Chem. 2007;282:20933-49.

14. Agre P, King LS, Yasui M, Guggino WB, Ottersen OP, Fujiyoshi Y, et al. Aquaporin water channels - from atomic structure to clinical medicine. J Physiol. 2002;542(Pt 1):3-16.

15. Zhu F, Tajkhorshid E, Schulten K. Theory and simulation of water permeation in aquaporin-1. Biophys J. 2004;86(1):50-7.

16. Sui H, Han B-G, Lee JK, Walian P, Jap BK. Structural basis of water specific transport through the AQP1 water channel. Nature. 2001;414:872-8.

17. Saparov SM, Kozono D, Rothe U, Agre P, Pohl P. Water and ion permeation of aquaporin-1 in planar lipid bilayers. J Biol Chem. 2001;276(34):31515-20.

18. Yool AJ, Weinstein AM. New roles for old holes: ion channel function in aquaporin-1. News Physiol Sci. 2002;17(2):68-72.

19. Kong $Y$, Ma J. Dynamic mechanisms of the membrane water channel aquaporin-1 (AQP1). Proc Natl Acad Sci USA. 2001:98(25):14345-9.

20. Ren G, Reddy VS, Cheng A, Melnyk P, Mitra AK. Visualization of a water selective pore by electron crystallography in vitreous ice. Proc Natl Acad Sci USA. 2001;98(4):1398-403.

21. de Groot BL, Frigato T, Helms V, Grubmuller H. The mechanism of proton exclusion in the aquaporin-1 water channel. J Mol Biol. 2003;333(2):279-93.

22. Heymann JB, Engel A. Aquaporins: phylogeny, structure, and physiology of water channels. News Physiol Sci. 1999;14(5):187-93.

23. de Groot BL, Grubmuller H. Water permeation across biological membranes: mechanism and dynamics of aquaporin-1 and GlpF. Science. 2001; 294(5550):2353-7.

24. Murata K, Mitsuoka K, Hirai T, Walz T, Agre P, Heymann JB, et al. Structural determinants of water permeation through aquaporin-1. Nature. 2000; 407(6804):599-605.

25. Wang $Y$, Tajkhorshid E. Molecular mechanisms of conduction and selectivity in aquaporin water channels. J Nutr. 2007;137:1509S-15S

26. Au CG, Cooper ST, Lo HP, Compton AG, Yang N, Wintour EM, et al. Expression of aquaporin 1 in human cardiac and skeletal muscle. J Mol Cell Cardiol. 2004;36:655-62.

27. Day RE, Kitchen P, Owen DS, Bland C, Marshall L, Conner AC, et al. Human aquaporins: regulators of transcellular water flow. Biochim Biophys Acta. 2014;1840(5):1492-506.

28. Martínez JL, Carrión A, Florián ME, Martín JA, López-Taylor JR, Fahey TD, et al. Aquaporin-1 gene DNA variation predicts performance in hispanic marathon runners. Medicina Sportiva. 2009;13(4):251-5.

29. Rivera MA, Martinez JL, Carrion AA, Fahey TD. AQP-1 association with body fluid loss in 10-km runners. Int J Sports Med. 2011;32(3):223-9.

30. Saunders CJ, Posthumus M, O'connell K, September AV, Collins M. A variant within the AQP1 $3^{\prime}$-untranslated region is associated with running performance, but not weight changes, during an Ironman Triathlon. J Sports Sci. 2015;33(13):1342-8.
31. Huang $Y-C$, Wang J-S. Effects of interval and continuous exercise regimens on aquaporin-1-mediated osmotic fragility of erythrocyte underhypoxic stress. FASEB Journal. 2016;30(1):S1240-6.

32. Xu L, Zhou Y, Courtney NA, Radfor TS, Boron WF. Effect of AQP1 knock out on mouse exercise tolerance. FASEB Journal. 2010;24(1):S609-4.

33. Alberrani V, De Castro Pietrangeli P, Rossi Mazza AM. The use of grey literature in health sciences: a preliminary survey. Bull Med Libr Assoc. 1990; 78(4):358-63.

34. Grzybowska EA, Wilczynska A, Siedlecki JA. Regulatory functions of 3'utrs. Biochem Biophys Res Commun. 2001;288(2):291-5.

35. Conne B, Stutz A, Vassalli J-D. The 3' untranslated region of messenger RNA: a molecular 'hotspot' for pathology? Nat Med. 2000;6(6):637-41.

36. Bouchard C. Adaptation to acute and regular exercise: from reductionist approaches to integrative biology. Prog Mol Biol Transl Sci. 2015;135:1-15.

37. Miyamoto-Mikami E, Zempo H, Fuku N, Kikuchi N, Miyachi M, Murakami H. Heritability estimates of endurance-related phenotypes: a systematic review and meta-analysis. Scand J Med Sci Sports. 2018;28(3):834-45.

38. Fábrega E, Berja A, García-Unzueta MT, Guerra-Ruiz A, Cobo M, López M, et al. Influence of aquaporin-1 gene polymorphism on water retention in liver cirrhosis. Scand J Gastroenterol. 2011:46:1267-74.

39. Wilber RL, Pitsiladis Y. Kenyan and Ethiopian distance runners: what makes them so good? Int J Sports Physiol Perform. 2012:7(2):92-102.

40. National Center for Biotechnology Information. Reference SNP cluster report rs1049305. 2018 [Available from: https://www.ncbi.nlm.nih.gov/projects/ SNP/snp_ref.cgi? rs =1049305\#Diversity.

41. Rüst CA, Knechtle B, Knechtle P, Wirth A, Rosemann T. Body mass change and ultraendurance performance: a decrease in body mass is associated with an increased running speed in male 100-km ultramarathoners. J Strength Cond Res. 2012;26(6):1505-16.

42. Butler TL, Au CG, Yang B, Egan JR, Tan YM, Hardeman EC, et al. Cardiac aquaporin expression in humans, rats, and mice. Am J Physiol Heart Circ Physiol. 2006;291(2):H705-13.

43. Frigeri A, Nicchia GP, Balena R, Nico B, Svelto M. Aquaporins in skeletal muscle: reassessment of the functional role of aquaporin-4. FASEB Journal. 2004;18(7):905-7.

44. Wakayama Y. Skeletal muscle regeneration may be enhanced by overexpression of aquaporin 1 in intramuscular capillary endothelial cells. Med Hypotheses. 2007;68(4):856-9.

45. Chandrasekar M, Suresh S, Srinivasan R, Bose AC. New analytical models to investigate thermal conductivity of nanofluids. J Nanosci Nanotechnol. 2009, 9(1):533-8.

46. Yool AJ. Functional domains of aquaporin-1: keys to physiology, and targets for drug discovery. Curr Pharm Des. 2007;13(31):3212-21.

\section{Publisher's Note}

Springer Nature remains neutral with regard to jurisdictional claims in published maps and institutional affiliations.

\section{Submit your manuscript to a SpringerOpen ${ }^{\circ}$ journal and benefit from:}

- Convenient online submission

- Rigorous peer review

- Open access: articles freely available online

- High visibility within the field

- Retaining the copyright to your article

Submit your next manuscript at $\boldsymbol{\nabla}$ springeropen.com 